\title{
Investigation of $\mathrm{ArF}^{*}$ excimer laser VUV radiation action on sapphire
}

\author{
S.M. Baschenko', K.S. Gochelashvili², R.M. Zakirov², V.I. Klimov², V.T. Mikhkelsoo ${ }^{3}$, \\ O.M. Prokhorov². \\ 1 - Institute of Physics, NAS of Ukraine, 46 Prospect Nauky, 03039 Kyiv, Ukraine \\ 2 - Institute of General Physics, RAS, 38 Vavilova str., 119991 Moscow, Russia \\ 3 - Institute of Physics, AS of Estonia, Tartu, Estonia
}

\begin{abstract}
Aluminium components of products escaped from the surface of a sapphire target being illuminated by VUV emission of an ArF* laser are investigated using the method of laser-induced fluorescence combined with the transient time technique. Relative concentrations of aluminium atoms and ions as well as their distribution on velocities in the flow are measured. Determined is also the temperature and velocity of aluminium atoms. It is ascertained that within the range of used energy densities of 0.45 to $1.0 \mathrm{~J} / \mathrm{cm}^{2}$ extraction of materials can be made in three regimes, namely: the ablation, the plasma and mixed ones, intertransitions between them being of a cyclic character.
\end{abstract}

Keywords: photoablation, sapphire, laser-induced fluorescence, $\mathrm{ArF}^{*}$ laser, $\mathrm{XeCl}^{*}$ laser complex.

Paper received 24.09.01; revised manuscript received 27.11.01; accepted for publication 12.12.01.

\section{Introduction}

Permanent growth and spread of using non-metallic super-hard and transparent within a wide spectral range materials, in particular for micro- and nanotechnologies, make the task of applying them to non-contact high-precision and fine processing by laser beam, especially in the non-thermal photoablation mode, to be important and timely. The most common and important differences of the photoablation mode against thermal evaporation are the following:

- minimum necessary influence of radiation on material extracted;

- minimum possible influence of radiation on the structure of the near-boundary material remained.

Fulfillment of these two conditions can provide maximum precision and minimum possible dimensions of a processed field with absence of melted material drops around the operation area as well as maximum smoothness of handled surfaces.

Radiation of short-wave excimer lasers (EL) is capable to initiate photoenhanced removal, extraction, ablation, mechanism of which can be principally distinguished from the mechanism of thermal evaporation (outburst): there exists a possibility to stimulate electron excitation of surface layer atoms into repulsive or weakly bound states due to EL emission, which results in the direct break of bonds and material removal extraction, ablation from the surface [1].

Among materials used in laser techniques, microelectronics, micro- and nanotechnologies, various refractory materials, in particular sapphire, find more and more wide applications. Investigations of morphology after sapphire surface destruction as a result of EL radiation action has shown that, under definite conditions, the melted material outburst due to hydrodynamic effects and descrepitation of the surface can be absent $[2,3]$. Results of investigations using laser-induced fluorescence (LIF) method for ascertaining energy distribution of sapphire ablation products arising under EL emission action evidence in favor of the non-thermal mechanism of photoevaporation [1].

As optimum and necessary processing regimes are mainly realized, as a rule, in a definite range of laser radiation parameters, one of important aspects of material laser processing is ascertaining and controlling interaction modes, in particularly, when approaching to a destruction thresh- 


\section{S.M.Baschenko et al.: Investigation of ArF* excimer laser VUV radiation...}

old of material. To far and wide understand the processes that take place, when the radiation interacts with materials, it is a most desirable to perform simultaneous control for the state of the sample-target processed and products that are extracted from this sample as well as processes taking place in these objects.

With this aim, we prepared and carried out detail investigations of the photoextraction process in sapphire, which was stimulated by $\mathrm{ArF}^{*}$ EL radiation with the wavelength $193 \mathrm{~nm}$. In the course of these investigations, we searched the interaction modes that provide effective photoablation of sapphire, i.e., realized is the interaction mechanism capable to promote removal of all atoms from the surface and to provide the highest purity of a processed surface.

\section{Experimental set}

As an object of investigations we used products escaping from EL-illuminated sapphire surface, in particular, aluminium atoms and ions. To study and determine their characteristics, we applied the method of laser-induced fluorescence in combination with the time-transient technique.

LIF spectrometer served as a base of our experimental set, its scheme is represented in Fig. 1. The main units of the LIF spectrometer are: an interaction chamber with necessary windows and a vacuum post, the forcing $\mathrm{ArF}^{*}$ laser, the source of resonance sounding radiation, that is $\mathrm{XeCl}^{*}$ excimer laser complex, and the system for registration of LIF signals.
In the chamber, the unit of sample fixation that provides three linear and one angle degrees of freedom was mounted. The way of the sounding beam was fixed. To reduce the level of scattered radiation, the sounding beam passed through a system of blackened diaphragms in the chamber. The vacuum post provided pressures of no more than $10^{-4}$ Torr in the interaction chamber.

As an forcing laser, we used an electrical discharge excimer ArF* laser (ELI-72) with the emission wavelength $193 \mathrm{~nm}$, pulse duration $12 \mathrm{~ns}$ and pulse energy $70 \mathrm{~mJ}$. As a source of sounding resonance radiation, we used $\mathrm{XeCl}^{*}$ laser complex with the wavelength smoothly tuned in the range of 307. $-308.4 \mathrm{~nm}$. The linewidth of radiation was $0.002 \mathrm{~nm}$.

The LIF signal registration system consisted of the optical line for collection of fluorescent light with the geometrical efficiency $0.8 \%$ and the monochromator MDR-2 (aperture is equal to $F / 2,5$ ). An optical signal from the monochromator output was registered with photomultiplier FEU-106 and then through the wideband amplifier U3-33 was transferred to an oscilloscope with memory and plotter. The time response of the registration system was $30 \mathrm{~ns}$.

\section{Experimental method}

Optically polished sapphire plates with $20 \times 5 \times 1 \mathrm{~mm}^{3}$ dimensions were placed into the vacuum chamber and irradiated by focused radiation of the ArF* excimer laser. Under its action, the irradiated area was partly removed. Via the small flighted-off substance cloud above sapphire surface, at a

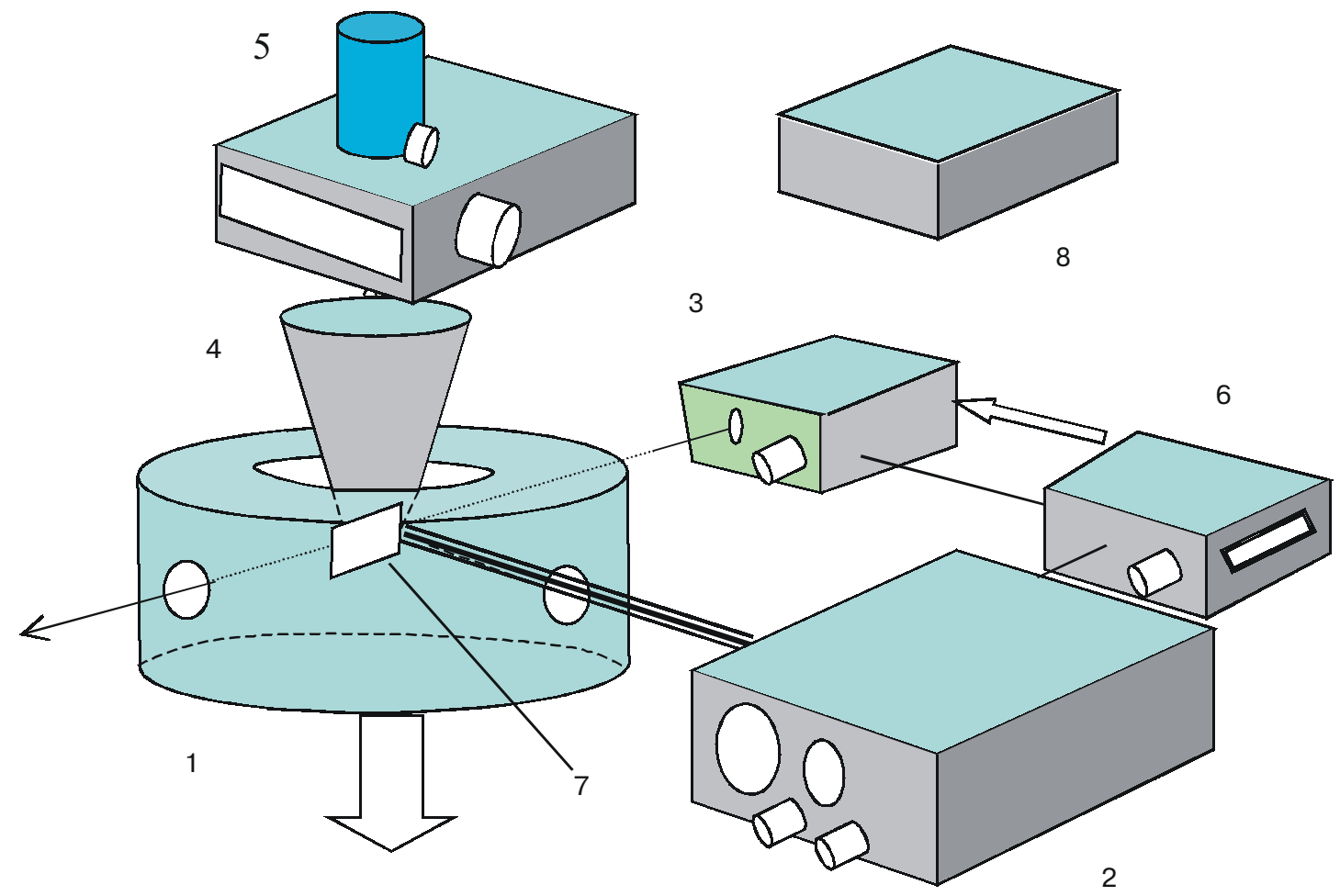

Fig. 1. Scheme of the experiment for studying sapphire ablation using the LIF method: 1 - interaction chamber; 2 - evaporating excimer $\mathrm{ArF}^{*}$ laser; 3 - $\mathrm{XeCl}^{*}$ excimer laser complex with tuned wavelength; 4 - LIF signal; 5 - monochromator of the optical system for LIF signal registration; 6 - time tuning unit; 7 - sapphire sample, 8 - registration system. 
definite time moment and at given distance from the surface, we directed the sounding radiation beam that could excite fluorescence of definite materials in that cloud. The receiving optical system collected this fluorescent signal and registered it. Geometry of this experiment was of the sort that the beam of forcing radiation was perpendicular to the sapphire surface, while the axis of the sounding beam and that of the system for a fluorescent signal collection were chosen parallel to the surface of the sample target and orthogonal to each other (Fig. 2). All three axes cross in the same point distantiated from the sample surface by $5 \mathrm{~mm}$. Such X-Y-Z- $\Delta$ geometry (with some shift $\Delta$ relatively to the surface) provides maximum possible spatial separation of the forcing and sounding beams as well as the LIF signal.

From the beam of forcing laser, cut by a diaphragm was a central homogeneous part with dimensions $20 \times 4 \mathrm{~mm}^{2}$ that was focused by the lens with focus distance $F=300 \mathrm{~mm}$ onto the target as a spot of $5 \times 1 \mathrm{~mm}^{2}$. The spot was oriented parallely to the sounding beam. To change the value of radiation flux that fell onto the sample, we used plates made of KU-1, KU-2, KV, KI sorts of silica and calibrated by their transmission. They were mounted one after another on the way of forcing beam to the focusing lens.

The time-transient method was realized by tuning a time delay between forcing and sounding pulses. It is noteworthy that the time-transient method automatically provides time separation of both forcing and sounding beams from LIF signals.

The sounding beam of our laser complex passed at the distance of $5 \mathrm{~mm}$ from the surface of sapphire sample-target and had dimensions $0.5 \times 1 \mathrm{~mm}^{2}$. The fluorescence signal was collected from the volume $0.5 \times 1 \times 12 \mathrm{~mm}^{3}$ (Fig. 3). The input slit of the monochromator was parallel to the sounding beam and the dimension of $12 \mathrm{~mm}$.

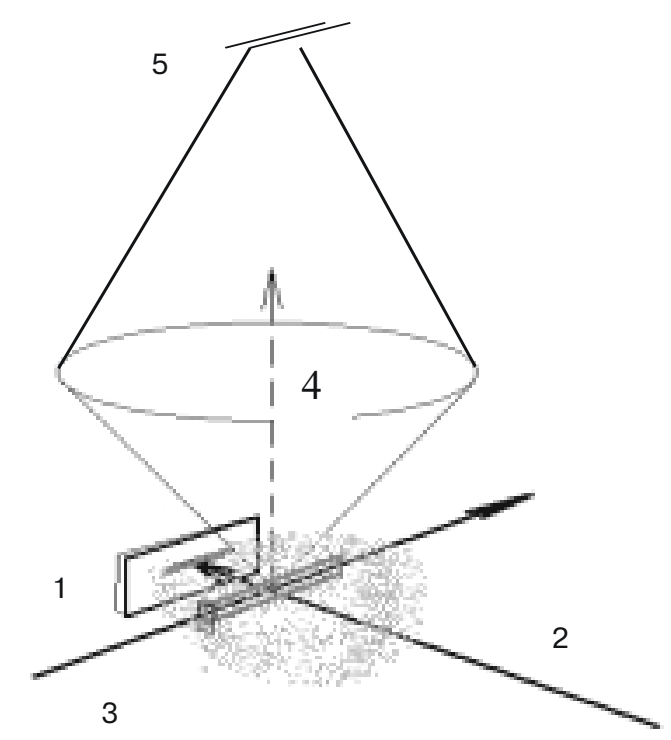

Fig. 2. Mutual spatial positions and orientation: 1 - sapphire sample; 2 - evaporating beam; 3 - sounding beam and 4 - spatial angle of LIF signal collection at the monochromator slit.

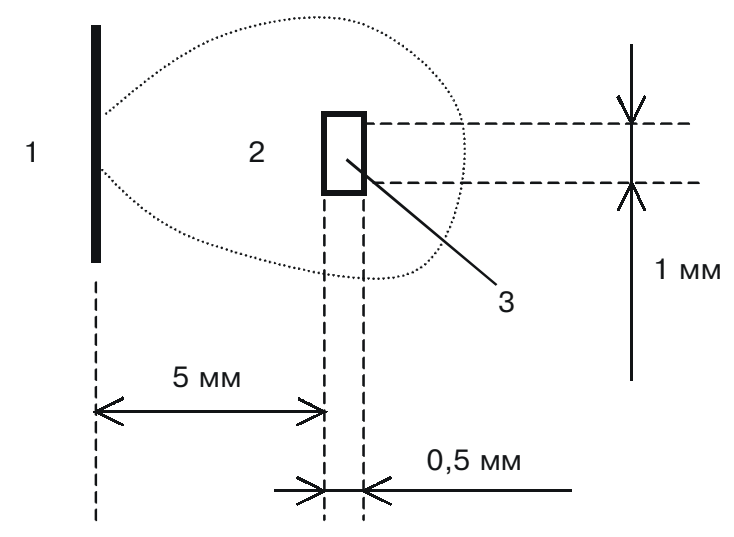

Fig. 3. Geometry of sounding: 1 - sample; 2 - evaporated cloud; 3 - cross-section of the sounding beam.

Sounding radiation of the $\mathrm{XeCl}{ }^{*}$ EL complex was tuned to the transition of the $\mathrm{Al}$ atom from the ground state $3 \mathrm{p}^{2}$ $P_{1 / 2}$ into the excited state $3 \mathrm{~d}^{2} \mathrm{D}_{3 / 2}$, which is corresponded to the wavelength $308.216 \mathrm{~nm}$ [4], while the LIF signal was registered using the transition $3 \mathrm{~d}^{1} \mathrm{D}_{3 / 2}-3 \mathrm{p}^{2} \mathrm{P}_{3 / 2}$ that is corresponded by the wavelength $309.284 \mathrm{~nm}$. In such manner we could reach also a spectral separation of the sounding radiation and LIF signals. The power of our EL complex was absolutely sufficient for saturation of this transition, which, in its turn, excluded any possible influence of sounding emission power fluctuations on the value of LIF signals. To precisely tune the wavelength of a sounding radiation, we used the optogalvanic effect [5] and respective lamp with hollow cathode LSP-1(Al).

It should be especially emphasized that such a tripletime, spatial and spectral-i.e. maximum possible detuning of the useful LIF signal from those of forcing and sounding beams provides high selectivity and sensitivity of the LIF method as well as its applicability for detection of impurities with extraordinary low relative concentrations down to $10^{-10}-10^{-12}$ on the background of other components with considerably higher concentrations [6-9].

It is obvious that the value of the LIF signal is proportional to the density of $\mathrm{Al}$ atoms being in the ground state $3 p^{2} \mathrm{P}_{1 / 2}$ at a given distance from the sample surface and in the definite moment of time. Taking into account that sounding was carried out at the distance $5 \mathrm{~mm}$, and characteristic velocities of product fligth-off are equal to $10^{6} \mathrm{~cm} / \mathrm{s}$, it is reasonable to state that even if $\mathrm{Al}$ atoms leave the sample surface when being in excited states, a lifetime of which is of the order of $100 \mathrm{~ns}$ [4], then they emit only in the thin near-surface layer before reaching the place where they should be detected. This place is reached by them in the ground non-excited $3 p$-state in definite time (approximately $5 \mathrm{~ms}$ )

But neutral $\mathrm{Al}$ atoms can be also produced as a result of electron-ion recombination processes. There arises the question: how can they be distinguished from those started from the sample surface in the neutral form? There is some opportunity for that. If one takes into account the fact that the time of electron-ion recombination characteristic for 


\section{S.M.Baschenko et al.: Investigation of ArF* excimer laser VUV radiation...}

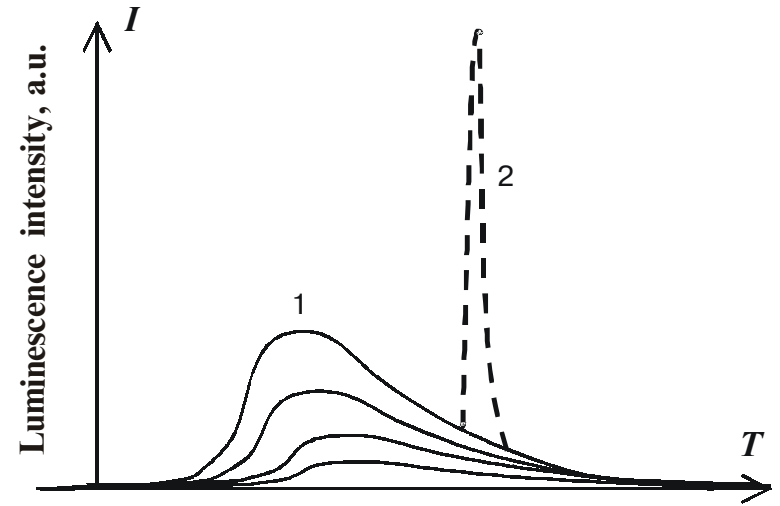

Time, a.u.

Fig. 4. Typical appearances of optical signals with the wavelength $309.284 \mathrm{~nm}$ : 1 - from plasma and 2 - atomic components of the flow.

given experimental conditions is about several microseconds [10], and the lifetime of the excited 3d-state for $\mathrm{Al}$ atoms is much less, then one can understand that the resulting signal can consist of two decaying exponents: from neutral (atoms) and plasma (ions) components - but with a large difference in decay times for luminescence signals (Fig. 4). It is the possibility that was used.

\section{Experimental results}

First signals evidencing upon the beginning of material removing, extraction, ablation from an optically polished sapphire surface started with the $\mathrm{ArF}^{*}$ laser radiation flux density close to $0.8 \mathrm{~J} / \mathrm{cm}^{2}$. In this case, observed was simultaneous appearance of signals from both ion (plasma) and atomic (ablation) components of the flow. In Fig. 5 shown is the way of reaching stationary values inherent to signals of ion and atomic components in dependence on the number of radiation pulses, when flow density is equal to $0.8 \mathrm{~J} / \mathrm{cm}^{2}$.

Signal stationary values are reached within $30-40$ pulses. As seen, at first, one can observes fast increase of the signal that is changed by a small drop when reaching the stationary value. It is obvious that this number of radiation pulses is sufficient both for cleaning the sapphire surface from technological products of its processing and removal of the so-called Griffith surface layer [11] that was subjected to structure changes when processed. And only af-

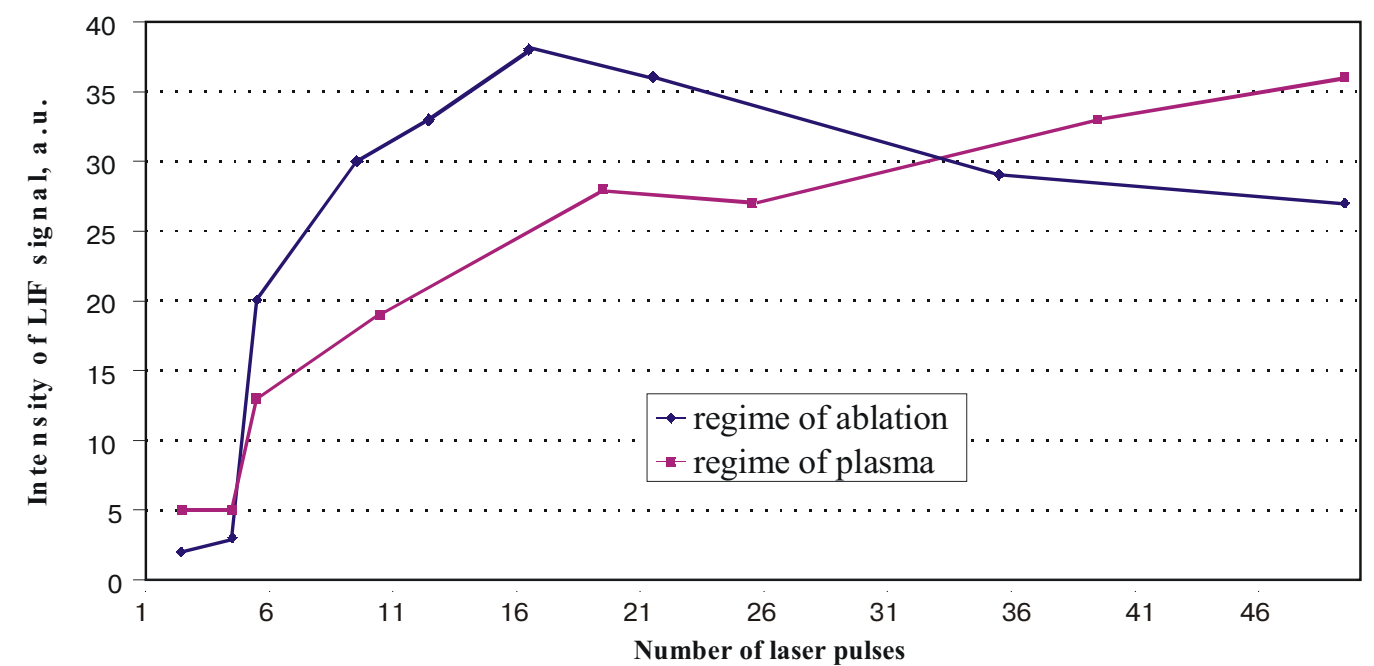

Fig. 5. Dependencies of LIF signals for atomic (1) and ion (2) flow components on the pulse number.

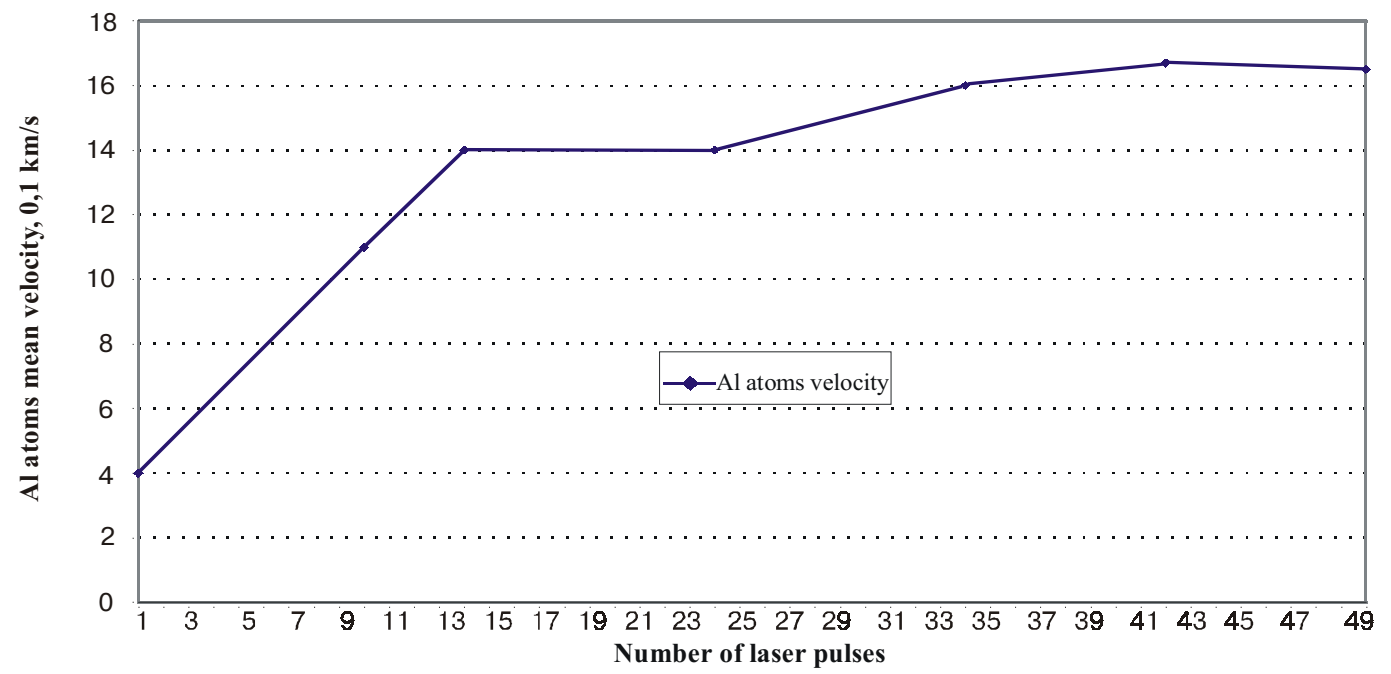

Fig. 6. Dependency of the mean $\mathrm{Al}$ atom velocity on the pulse number. 
ter that, radiation begins to interact with bulk material nondestroyed when being processed. At this stage, the ratio of ionic to atomic components increases. Besides, with this growth of an ionic component share, one can observe the increase of the mean velocity of $\mathrm{Al}$ ion escape from the sample surface in the range of $9 \cdot 10^{5}$ up to $1.7 \cdot 10^{6} \mathrm{~cm} / \mathrm{s}$, which is depicted in Fig. 6.

After cleaning the sapphire surface from technological material remains and removing the destroyed layer as well as reaching the stationary regime by the LIF signal at the flux density $0.8 \mathrm{~J} / \mathrm{cm}^{2}$, one can observe a transition into the mode of predominant plasma (ion) removal, which is represented by the upper plot in Fig. 7. It is worth to note that the plasma removal mode takes place not only at densities larger than $0.8 \mathrm{~J} / \mathrm{cm}^{2}$ but at the less ones, too. With increasing flux density in this regime, the LIF signal is increased both from neutral (atomic) and plasma (ionic) components of the flow. It means that removed material amount grows. With changing the flux, the relative part of the plasma (ionic) component is changed, too (Fig. 8).

When fluxes are less than $0.45 \mathrm{~J} / \mathrm{cm}^{2}$, stationary LIF signals are observed within approximately 20 pulses and then decrease quickly (within 3-4 pulses) down to zero. Hereafter, when using such energy densities, we observed neither ablation nor plasma component signals.

After slowing down the plasma regime, when the flow density grows up to $0.5 \mathrm{~J} / \mathrm{cm}^{2}$, the interaction becomes consistent with the ablation regime presented by the lower plot

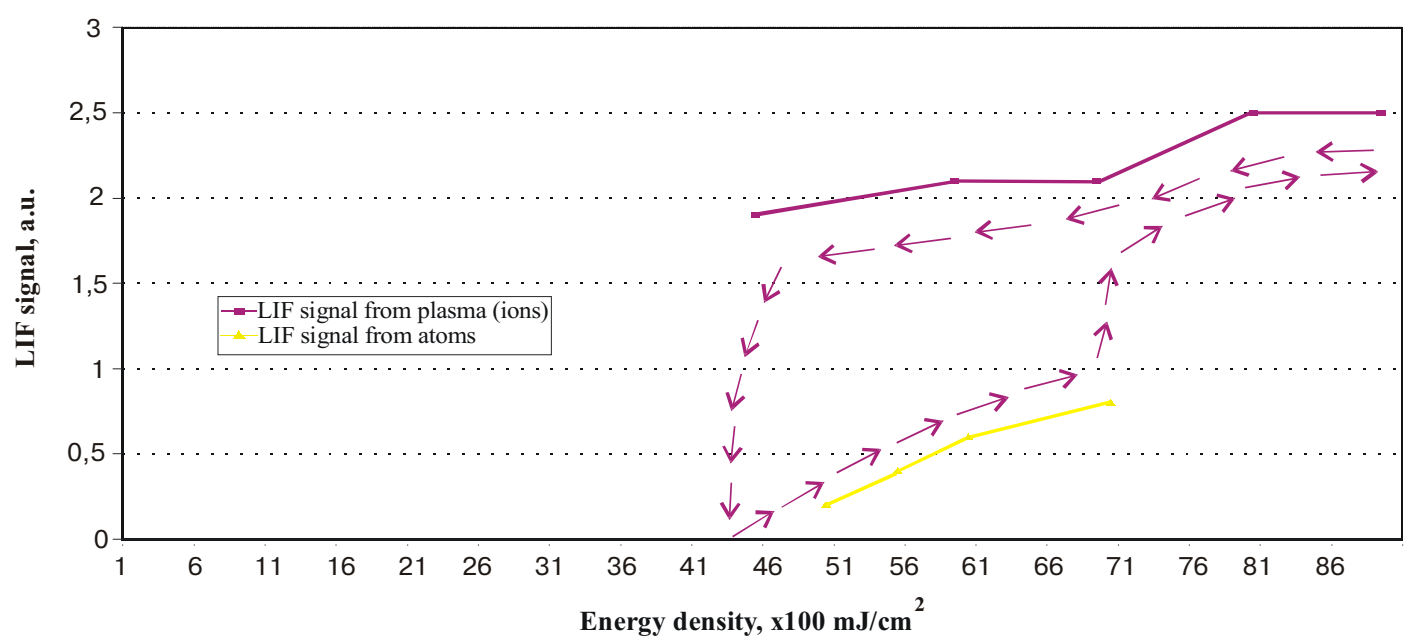

Fig. 7. Dependencies of LIF signal magnitudes on the emitted energy density for plasma and ablation flow components and their mutual relation.

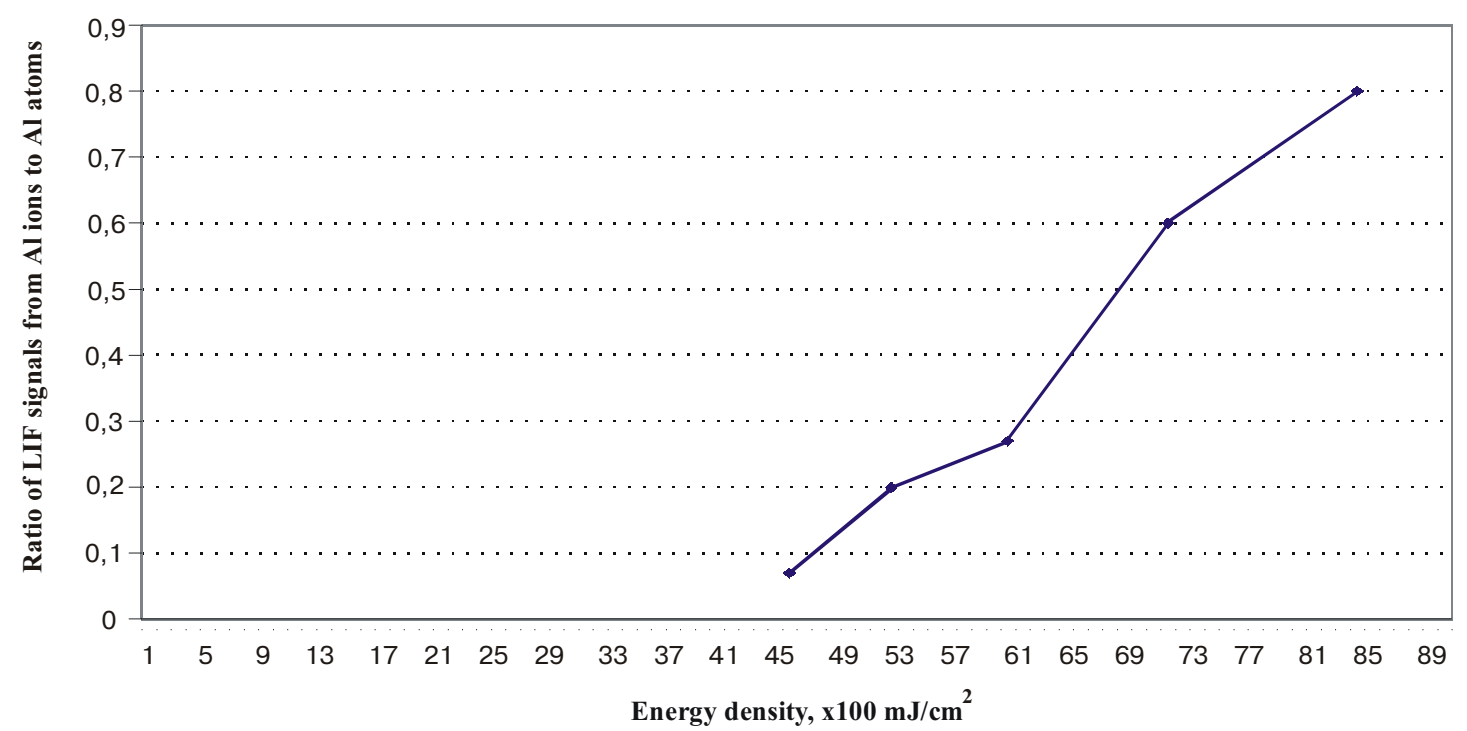

Fig. 8. Dependency of the relative share of the LIF signal plasma component on the emitted energy density during a transition into the plasma regime of evaporation. 


\section{S.M.Baschenko et al.: Investigation of ArF* excimer laser VUV radiation...}

in Fig. 7. During this regime, in flighting out clouds, we can observe only the neutral atomic, namely ablation, component of the flow. Evaporated material amount increases with increasing the flow density in this regime. The ablation regime (of removing) takes place in the range of energy flow densities of 0.5 to $0.7 \mathrm{~J} / \mathrm{cm}^{2}$ and is characterized by less material removal from sapphire surface in comparison with the plasma regime.

When the flow density reaches the value $0.7 \mathrm{~J} / \mathrm{cm}^{2}$, a transition into the plasma regime of interaction and material removing takes place. This regime is characterized by rapid increase of the signal inherent to the plasma component of the flow and the respective growth of the LIF signal by 2.5-3 times. Attaining stationary values has similar features like those characteristic for cleaning the surface (Fig. 5) but can be realized within less number of pulses (approximately twenty).

To return into the ablation regime of interaction, it is necessary: 1) to reduce the flow density to the value less than $0.45 \mathrm{~J} / \mathrm{cm}^{2}$, in doing so signals from ion and atomic components disappear within 20 pulses, and then 2) to raise the flow density up to the value higher than $0.5 \mathrm{~J} / \mathrm{cm}^{2}$. Thus, influencing upon sapphire surface with $\mathrm{ArF}^{*}$-laser radiation in the range of density fluxes from 0.5 to $0.7 \mathrm{~J} / \mathrm{cm}^{2}$ we can simultaneously realize both plasma and ablation regimes of interaction with an important opportunity of cyclic transitions from one regime to another.

In Fig. 9 shown is the distribution of $\mathrm{Al}$ atoms upon velocities of escape from the surface measured by the timetransient method in the ablation regime when the flux density is $0.65 \mathrm{~J} / \mathrm{cm}^{2}$. The maximum value of the LIF signal (and, respectively, the maximum concentration of $\mathrm{Al}$ atoms in the ablation cloud) is corresponded by the velocity $9 \cdot 10^{5} \mathrm{~cm} / \mathrm{s}$.

In Fig. 10 represented are data of the mean escape velocity for $\mathrm{Al}$ ions in the plasma regime of evaporation and Al atoms in the ablation one in dependence of the emission flux density. As seen, in the plasma regime, the escape velocity grows from $1.3 \times 10^{6}$ to $1.8 \times 10^{6} \mathrm{~cm} / \mathrm{s}$ with increasing the energy density from 0.45 to $0.8 \mathrm{~J} / \mathrm{cm}^{2}$. In its turn, in the ablation regime, the escape velocity of $\mathrm{Al}$ atoms remains approximately constant and equal to $8 \cdot 10^{5} \mathrm{~cm} / \mathrm{s}$ with changing energy density. It is worth to note the essential difference in escape velocities of $\mathrm{Al}$ atoms in the plasma regime and atoms in the ablation one within the range of energy

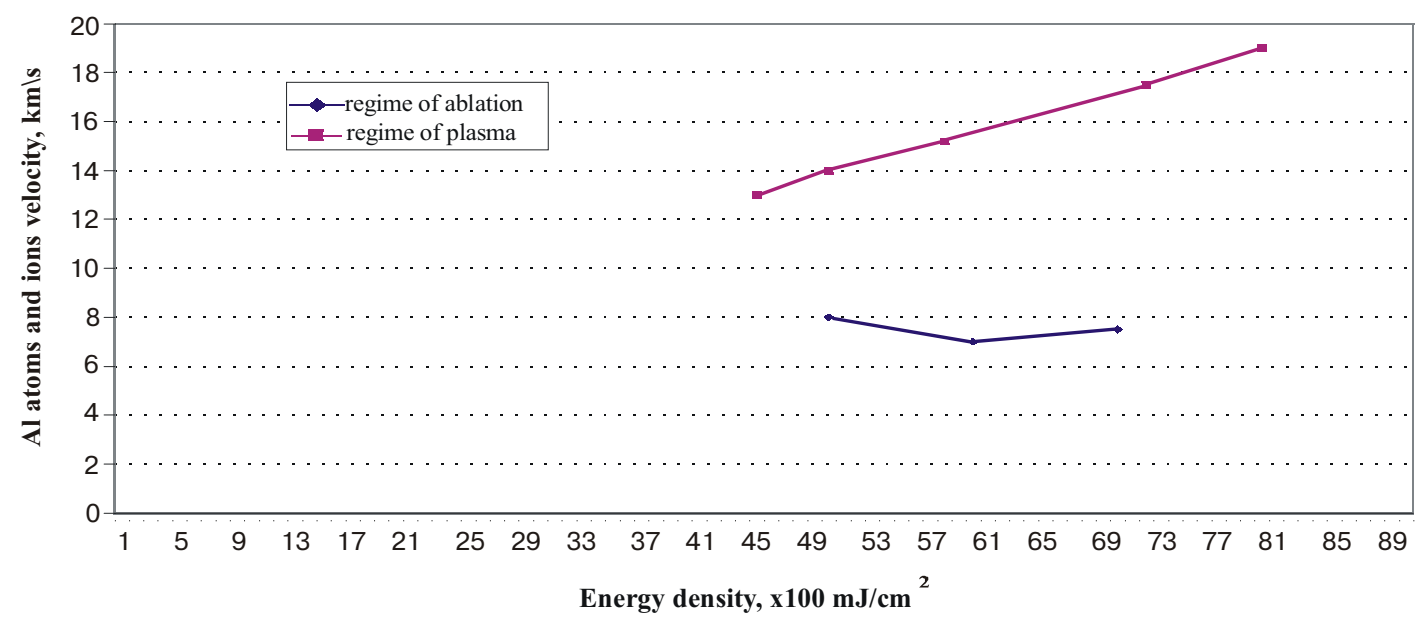

Fig. 9. Velocity distribution of $\mathrm{Al}$ atoms in the ablation flow when the density of incident emission energy is equal to $0.66 \mathrm{~J} / \mathrm{cm}^{2}$.

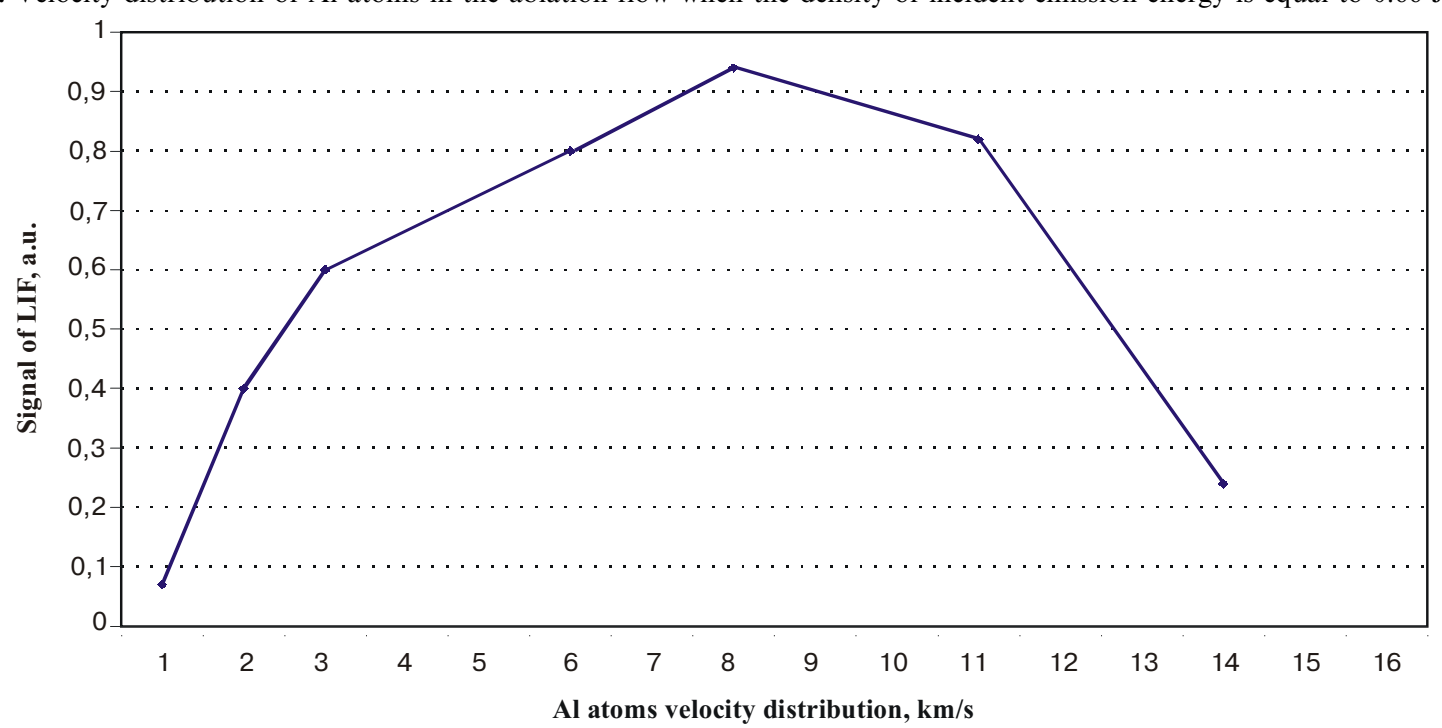

Fig. 10. Dependency of the $\mathrm{Al}$ atom mean velocity on the density of the emission energy incident onto the sample. 
S.M.Baschenko et al.: Investigation of ArF* excimer laser VUV radiation...

flux densities of 0.5 to $0.7 \mathrm{~J} / \mathrm{cm}^{2}$, where both regimes are realized simultaneously. In the plasma regime, when radiation fluxes have equal values, the escape velocity of ions is 1.5-2 times higher than that of atoms in the ablation one.

\section{Analysis of results obtained. Main criteria of realizing ablation and plasma regimes of interaction of radiation with sapphire}

Obtained results enable to separate and ascertain two essentially different regimes of $\mathrm{ArF}^{*}$-EL radiation interaction with sapphire surface, when energy flow are close to a threshold of destruction.

The plasma regime arises directly after beginning of radiation interaction with sapphire surface at the flux 0.8 $\mathrm{J} / \mathrm{cm}^{2}$ or as the transient from the ablation one at the flux 0.7 $\mathrm{J} / \mathrm{cm}^{2}$, and takes place even at fluxes decreasing down to $0.45 \mathrm{~J} / \mathrm{cm}^{2}$.

In the course of transition from the ablation regime into the plasma one at the flux $0.7 \mathrm{~J} / \mathrm{cm}^{2}$, we can observe the following characteristic features:

- the share of the plasma component in an escaping cloud is increased,

- the escape velocity of $\mathrm{Al}$ ions grows from $8 \cdot 10^{5}$ up to $1.8 \cdot 10^{6} \mathrm{~cm} / \mathrm{s}$, which corresponds to the change of the kinetic energy value approximately from 9 up to $45 \mathrm{eV}$,

- the amount of material removed from the surface is more than two-fold increased.

The increase of the $\mathrm{Al}$ ion velocity at the plasma regime can be explained by an action of the electrostatic acceleration mechanism [12] that results in appearance of atoms and ions with large kinetic energies near the surface [13].

The fact that with a decreasing energy flux down to 0.45 $\mathrm{J} / \mathrm{cm}^{2}$, signals from the ion component of the flow are observed within approximately 20 pulses and then rapidly (within 3-4 pulses) drop to zero, being non-observed further at these energy densities as well as in combination with sharply distinctive characteristics of escaping products under this interaction regime, can evidence in favor of creation of near-surface (plasma) layer with characteristics differing from that of main material. Estimating the material layer thickness extracted within one pulse as approximately $1 \mathrm{~nm}$ [1] and taking into account cessation of material extraction after 20 pulses, one can judge the thickness of this specific near-surface layer as some $20 \mathrm{~nm}$. Above dependence of $\mathrm{Al}$ ions on the energy flux can give an indication of a thickness dependence for this specific (plasma) layer on an energy flux.

In favor of the assumption about creation, existence and exhaustion of this specific near-surface layer in the plasma interaction regime, definite features similar to a hysteresis effect can betoken, too. Namely, this regime can: i) begin at the energy density $0.8 \mathrm{~J} / \mathrm{cm}^{2}$ that is higher than the upper limit of ablation regime existence $0.7 \mathrm{~J} / \mathrm{cm}^{2}$; ii) be sustained at the energy density $0.45 \mathrm{~J} / \mathrm{cm}^{2}$ that is below than the lower limit of ablation regime existence $0.5 \mathrm{~J} / \mathrm{cm}^{2}$; iii) stem from the ablation one, when the upper limit of the latter $0.7 \mathrm{~J} / \mathrm{cm}^{2}$ is exceeded.
The pure ablation regime is observed only in the range of flow densities from 0.5 to $0.7 \mathrm{~J} / \mathrm{cm}^{2}$ and only from the surface that was previously cleaned from technological products (it means that the layer disturbed as a result of mechanical processing was extracted) and only from the surface that has passed the cycle of withdrawing, exiting out of the plasma regime. In the absence of an ionic component, the electrostatic acceleration of escaping products does not take place, and the mean velocity of neutral $\mathrm{Al}$ atoms is less than that of ions and is equal roughly to $8 \cdot 10^{5}(9 \mathrm{eV})$. Contrary to the ion case, it does not depend on the emission flux (Fig. 10).

As the width of the sapphire forbidden gap is about $9 \mathrm{eV}$, then, at the quantum energy $6.4 \mathrm{eV}$, a respective electron excitation needs the two-photon absorption. It is the point that determines existence of the threshold value for the radiation flux that provides ablation beginning. For the wavelength $193 \mathrm{~nm}$, the threshold value is about $0.5 \pm 0.1$ $\mathrm{J} / \mathrm{cm}^{2}$, which is in logical accordance with that of the wavelength $248 \mathrm{~nm}$, namely, $0.6 \pm 0.1 \mathrm{~J} / \mathrm{cm}^{2}$ [3]. With an accuracy not exceeding measurement errors, we observed coincidence of radiation flux values that correspond with the beginning of plasma appearance, and, consequently, intervals where the ablation regime exists [14]. But in this case, there is an essential difference in velocities and, respectively, in kinetic energies of $\mathrm{Al}$ atoms that escape from the surface in the ablation regime: $4 \mathrm{eV}$ for $248 \mathrm{~nm}$ and $9 \mathrm{eV}$ for $193 \mathrm{~nm}$

In the plasma regime, the kinetic energy $20 \mathrm{eV}$ corresponds to the threshold flux $0.45 \mathrm{~J} / \mathrm{cm}^{2}$ at $\lambda=193 \mathrm{~nm}$. And at $\lambda=248 \mathrm{~nm}$, the same energy of Al atoms can be reached at much larger flux, namely, $3 \mathrm{~J} / \mathrm{cm}^{2}[3]$.

The main differences between ablation and plasma regimes for radiation of 193 and $248 \mathrm{~nm}$ wavelengths interaction with sapphire surface are as follows. The transition of the ablation regime into the plasma one at $\lambda=193 \mathrm{~nm}$, contrary to that for $\lambda=248 \mathrm{~nm}$, takes place with a simultaneous change of sapphire structure, as the plasma ignited can essentially influence on the sapphire surface. In this case, grown rapidly are both escaped material amount (more than two-fold) and the velocity of material escaping from the surface (by 1.5-2 times). At the wavelength $248 \mathrm{~nm}$, the similar influence of plasma on sapphire surface was not observed [14]. Formation of the specific sub-surface layer results also in a co-existence of plasma and ablation regimes at $193 \mathrm{~nm}$ within the range of overlapping flux intervals for the plasma mode (when the flux is more than 0.45 $\mathrm{J} / \mathrm{cm}^{2}$ ) and for the ablation one (for fluxes $0.5-0.7 \mathrm{~J} / \mathrm{cm}^{2}$ ), while the transition between them has a cyclic character, in contrast with a continuous transition at $248 \mathrm{~nm}$.

\section{Conclusions}

Using the method of laser-induced fluorescence, investigated has been the interaction of the $\mathrm{ArF}^{*}$ excimer laser radiation $(\lambda=193 \mathrm{~nm})$ with an optically polished sapphire surface. Ascertained was the existence of at least two different regimes for such interaction: the plasma and ablation ones. Determined were ranges of power densities corre- 


\section{S.M.Baschenko et al.: Investigation of $\mathrm{ArF}^{*}$ excimer laser VUV radiation...}

sponding to these regimes as well as performances and features of both regimes. It enabled us to demonstrate the possibility of applying the above method for clear distinguishing the ablation and plasma regimes of the radiation interaction with sapphire.

\section{References}

1. Dreyfus R.W., Kelly R., Walkup R.E. // Appl. Phys. Lett., 49, p. 1478 (1986).

2. Dreyfus R.W., Walkup R.E. // Rad. Eff., 99, p. 199 (1986).

3. Rothenberg J.E., Kelly R. // Nucl. Instr. and Meth. B1, p.291 (1984).

4. Handbook on atomic and molecular physics / A.A.Radtsig, B.M.Smirnov, "Atomizdat", Moscow, 1980.

5. King D., Schenk P., Smyth P. et al. // Appl. Opt., 16, p. 2617 (1977).

6. Tönnermann A., Mossavi K., Wellegehausen B. Nonlinear optic processes in the near-resonant two-photon excitation of xenon by femtosecond KrF-excimer-laser pulses // Appl. Phys. A46 (5), p.2707-2717 (1992).

7. On opportunity to determine metals in gases by the laser-induced fluorescence method (in Russian) // Zhurnal prikladnoi spektroskopii 51(3), p. 461-465.

8. Kelly R., Cuomo J.J., Leary P.A., Rothenberg J.E., Braren B.E., Aliotta C.F. // Nucl. Instr. and Meth. B9, p.329 (1985).

9. Dreier T., Dreizler A., Wolfrum J. The application of a ramanshifted tunable $\mathrm{KrF}^{*}$ excimer laser for laser-induced fluorescence combustion diagnostics // Appl. Phys. B. 55 (4), p.381387 (1992).

10. Kinetics of non-equilibrium low-temperature plasma, L.M.Biberman / Moscow, Nauka, 1982.

11. A.A.Griffith // Phil. Trans. Roy. Soc. London, A221, 163, 1920.

12. Bychkovski Yu.A., Degtyarenko N.N., Elesin V.F., Kondrashov V.E., Lovetski E.E. // Zhurnal tekhnicheskoi fiziki, XLIII, p. 2540 (1973).

13. Akhsakhalyan A.D., Bityurin Yu.A., Gaponov S.V., Gudkov A.A., Lugin V.I. // Zhurnal tekhnicheskoi fiziki, 52, p.1584 (1982).

14. Dreyfus R.W., Jasinski J.M., Walkup R.E., Kelly R. // CLEO-85, PQ2, 306. 